\title{
Aplicações Embarcadas em Sistemas Multiprocessadores: banco de aplicações para teste de técnicas de projeto incluindo descrição formal por grafo de tarefas, fluxo de informações e comportamento dinâmico.
}

\author{
Alexandre Ribeiro Lopes (IC), Alice M. Tokarnia (PQ).
}

\section{Resumo}

Muitos pesquisadores necessitam de um conjunto de aplicações para testar novas técnicas de projetos. Não é sempre possível encontrar um conjunto de aplicações que consiga atender aos requisitos do pesquisador. $O$ trabalho apresenta um conjunto de aplicações composto por sete aplicações para sistemas embarcados multiprocessador que são apresentados juntamente com a descrição das tarefas das aplicações em formato de grafos, informações sobre tempos de execução das aplicações e uma descrição da transmissão de dados e rastro de acesso à memória pelas tarefas paralelas.

Palavras Chave: Conjunto de aplicações, aplicações embarcadas, sistemas multiprocessador.

\section{Introdução}

Sistemas multiprocessadores propostos para conciliar desempenho e consumo adequado de energia vem sendo alvo de muitos trabalhos de pesquisa e desenvolvimento. Em particular, 0 desenvolvimento de novas técnicas de projeto é essencial para 0 uso eficiente de multiprocessadores em futuros sistemas embarcados. Conjuntos de aplicações de testes são muito importantes para a avaliação de algoritmos de projeto de sistemas embarcados e devem ser baseados em sistemas reais ( Hennesey and Patterson, 2011).

Este trabalho propõe a criação de um conjunto de aplicações de testes que venha atender às necessidades de pesquisadores.

\section{Resultados e Discussão}

Para este trabalho, obtivemos informações correspondentes a sete aplicações. Foram utilizadas as formas de paralelismo de dados e pipeline (Bienia and Li, 2010) nestas aplicações.

Os testes foram feitos utilizando 4 Gbytes de memória RAM e um processador i3-350M com 2 núcleos e 4 Threads físicas e i7-3770k com 8 Threads físicas.

Uma das aplicações utilizadas foi o Compressor JPEG. O algoritmo utilizado necessitou ser paralelizado. Para isso, utilizamos Pthreads (Posix Threads) e exploramos paralelismo de dados. A soma dos tempos de sistema e usuário, o tempo real e a soma dos tempos de usuário e sistema divididos pelo valor mínimo entre o número de threads e threads físicas utilizando uma matriz de entrada que representa uma imagem de $1280 \times 2560$ pixels podem ser vistas na Figura 1.

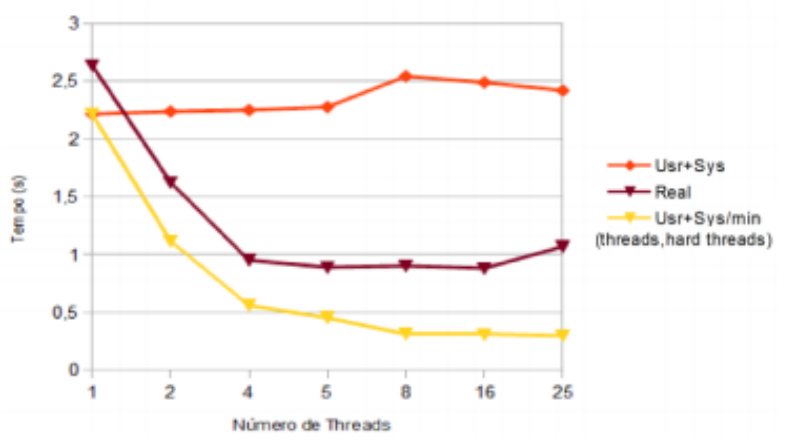

Figura 1. Inserir aqui título de figura.

\section{Conclusões}

O trabalho propõe a criação de um conjunto de aplicações de testes para atender às necessidades de pesquisadores em avaliar algoritmos para projetos de sistemas embarcados. Propomos uma análise dos algoritmos buscando também levantar algumas informações adicionais. O trabalho conta com sete aplicações com mais de um tipo de paralelismo e mais de uma área.

\section{Agradecimentos}

Agradeço a minha orientadora Alice e colegas do LCA. Pesquisa financiada pelo PIBIC/CNPQ.

C. Bienia and K. Li. Characteristics of workloads using the pipeline programming model. In Proceedings of the EAMA'10, Jun. 2010. J. L. Hennesey and D. A. Patterson. Computer Architecture : A Quantitative Approach. Kaufmann Publishers, Inc., San Francisco, CA., 5th edition, 2011. 